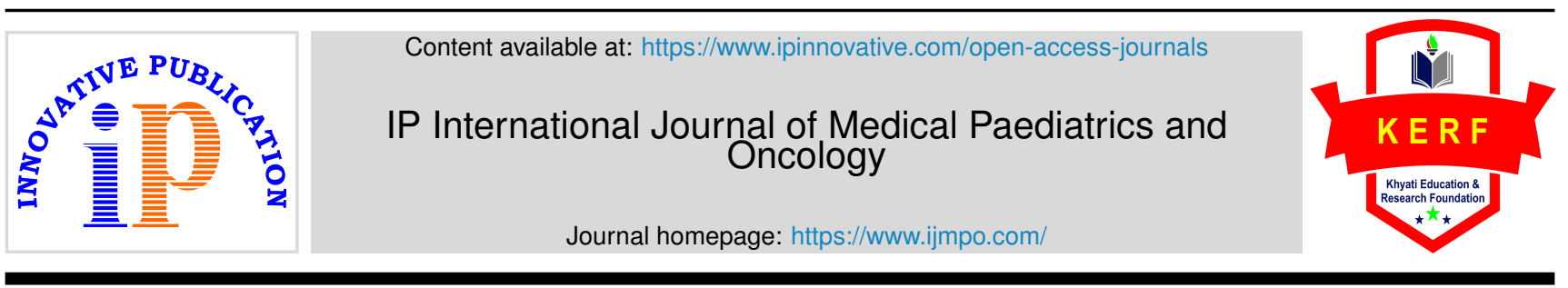

Original Research Article

\title{
A prospective study on etiological factors and clinical indicators in term and near term neonates admitted with hyperbilirubinemia in nicu in a tertiary care hospital in Hyderabad, India
}

\author{
Syed Adnan Ali ${ }^{1}$, C.V.S. Lakshmi1 ${ }^{1}$, Uppin Narayan Reddy ${ }^{2}$, Farhana Nazneen $^{1}$, \\ Muzammil $^{1}$ \\ ${ }^{1}$ Dept. of Pediatrics, Princess Esra Hospital, Deccan College of Medical Sciences, Hyderabad, Telangana, India \\ ${ }^{2}$ Dept. of Pediatrics, Deccan College of Medical Sciences, Hyderabad, Telangana, India
}

\section{A R T I C L E I N F O}

\section{Article history:}

Received 15-05-2021

Accepted 18-06-2021

Available online 03-08-2021

\section{Keywords:}

BIND (Bilirubin induced neurological damage)

DVET (Double Volume Exchange

transfusion)

Neonatal hyperbilirubinemia

Physiological jaundice

blood group incompatibility

\begin{abstract}
A B S T R A C T
Objectives: 1 . To study the etiology and risk factors of neonatal hyperbilirubinemia in term and near-term infants. 2. To study the clinical course of these infants during NICU stay

Materials and Methods: The present study was conducted at NICU, Department of Pediatrics, Princess Esra hospital, Deccan Medical College, Hyderabad, India, from October 2019 to October 2020. Term and late preterm infants admitted in NICU with Serum Bilirubin levels more than $12 \mathrm{mg} / \mathrm{dl}$ were included in the study. The risk factors, etiology and clinical profile of these infants during NICU stay were studied.

Results: 210 neonates were admitted in NICU with hyperbilirubinemia (Serum Bilirubin $>12 \mathrm{mg} / \mathrm{dl}$ ) during the study period, out of which 118 were male $(56.20 \%)$ and 92 were female $(43.80 \%)$. Neonates were further distributed based on gestational age, in which 46 (21.90\%) were late pre-terms i.e. between 3437 weeks and 164 neonates $(78.10 \%)$ were full term i.e. greater than 37 weeks. The neonates were also classified based on their birth weight, with neonates between 2500-3000 grams having the highest incidence $(46.19 \%)$. Lastly, the etiological and risk factors were assessed and quantified, with physiological jaundice occurring as the major cause and late prematurity as the most common risk factor associated with neonatal hyperbilirubinemia. The average duration of phototherapy was 2.50 days and 3 babies required Double Volume Exchange Transfusion (DVET) for significant hyperbilirubinemia.

None of the babies requiring DVET had clinical features of Bilirubin Induced Neurological Dysfunction (BIND) during NICU stay.

Conclusion: 1. Most common cause of neonatal hyperbilirubinemia was found to be Physiological followed by Septicemia and Idiopathic etiologies. Blood group incompatibilities were less common causes. 2. Phototherapy is a cheap and effective way to reduce bilirubin levels in neonatal jaundice. 3. Exchange transfusion is a safe procedure and should be considered when indicated, to decrease the incidence of BIND. All cases requiring DVET were due to blood group incompatibility.

(C) This is an open access article distributed under the terms of the Creative Commons Attribution License (https://creativecommons.org/licenses/by/4.0/) which permits unrestricted use, distribution, and reproduction in any medium, provided the original author and source are credited.
\end{abstract}

\section{Introduction}

Hyperbilirubinemia is one of common problems seen in neonates requiring admission for phototherapy. ${ }^{1}$ Serum bilirubin concentration exceeding $5-7 \mathrm{mg} \%$ causes neonates to appear clinically icteric. ${ }^{2}$

\footnotetext{
* Corresponding author.

E-mail address: lakshmicvsdr@gmail.com (C. V. S. Lakshmi).
}

Jaundice is the commonest abnormal clinical finding in neonates. ${ }^{3}$ The average incidence of neonatal jaundice in India, as reported in several publications, is around 54.6\% to $77 \% .{ }^{4}$ Jaundice occurs when the immature neonatal liver is unable to clear a sufficient amount of bilirubin from the plasma. ${ }^{5}$ Yellowish discoloration of the skin is the result of accumulation of unconjugated lipid-soluble bile pigment. ${ }^{6}$ 
Pathological jaundice is known to occur in $4-8 \%$ of newborns. ${ }^{7}$ The common causes include blood group incompatibilities, prematurity, G6PD deficiency and sepsis with majority being idiopathic. Other less common causes include Infants of diabetic mothers, cephalhematoma and polycythemia. ${ }^{8}$ The immature brain of the newborn is susceptible to unconjugated bilirubin toxicity resulting in the acute manifestation, BIND. ${ }^{9}$

Assessment of jaundice should be done in the natural light. The pulp of the finger or thumb is pressed into the baby's skin, preferably over a bony part till it blanches and underlying skin is noted for the yellow discoloration.

The criteria for intervention to control hyperbilirubinemia vary in different clinical situations, and on gestational age of the infants. Intervention for neonatal jaundice is based on guidelines for phototherapy and exchange transfusion for infants more than 35 weeks of gestation as published by the American Academy of pediatrics. ${ }^{10}$ Phototherapy is a cheap, effective and safe method of management of neonatal hyperbilirubinemia. ${ }^{11}$

The present study will try to analyze the causes, risk factors, both maternal and neonatal, and clinical profile of neonates admitted with hyperbilirubinemia.

\section{Materials and Methods}

The present study was conducted at NICU, Department of Pediatrics, Princess Esra hospital, Deccan Medical College, Hyderabad, India, from October 2019 to October 2020. A written informed consent was obtained from all parents of the babies involved in this study.

\subsection{Selection of cases}

The babies delivered at the hospital as well as babies referred from other hospitals of age 0-28 days during a period of one-year duration were included in this study. Late pre term and full-term babies, both healthy and sick, were included.

\subsection{Sample size}

Comprised of 210 neonates with jaundice (serum bilirubin more than $12 \mathrm{mg} / \mathrm{dl}$ ) hospitalized in the neonatal unit in Princess Esra hospital during the study period.

\subsection{Sampling method}

Total of 210 hospitalized neonates satisfying selection criteria were recruited.

\subsection{Inclusion criteria}

1. Neonates with hyperbilirubinemia $>12 \mathrm{mg} / \mathrm{dl}$ admitted in NICU of Princess Esra hospital, Hyderabad during study period.
2. Both intramural and extramural babies were included in the study.

3. Age group between 0-28 days.

4. Infants more than $33+6$ weeks of gestation.

\subsection{Exclusion criteria}

1. Babies attending outpatient department only.

2. Babies who got discharged against medical advice.

3. Babies less than 34 weeks.

4. Those who did not give consent to participate in the study.

\subsection{Methods}

Jaundice was identified and assessed by clinical methods and confirmed by biochemical methods. Jaundice was clinically estimated by Kramer's rule in broad daylight and confirmed by Serum bilirubin. Detailed antenatal, natal and postnatal history was taken. Clinical risk factors for jaundice were documented for every baby, and a detailed neurological examination including BIND score was performed for all babies requiring treatment for jaundice. A basic evaluation for cause for hyperbilirubinemia (CBP, Reticulocyte count, G6PD and DCT) was done in babies with Serum bilirubin levels more than $18 \mathrm{mg} / \mathrm{dl}$.

Serum Bilirubin measurement estimation was done via Van den Bergh method. Only those babies having serum bilirubin concentration of more than $12 \mathrm{mg} / \mathrm{dl}$ were included in the study.

Babies were treated according to guidelines published by American Academy of Pediatrics charts with phototherapy and/or exchange transfusion. Serum bilirubin levels were monitored periodically. The neonates were treated according to standard guidelines and monitored throughout their stay in the hospital. The side effects of phototherapy and/or DVET were documented during NICU stay.

\subsubsection{Statistical analysis}

Using Microsoft word, Microsoft excels and Epi Info 7 Statistical analysis was done.

\subsubsection{Ethical approval}

Approval from Institutional review board was obtained before the study was initiated.

\section{Results}

In this study of 210 neonates, $118(56.20 \%)$ were male while $92(43.80 \%)$ neonates were female as shown in Figure 1.

Out of 210 neonates studied, $46(21.90 \%)$ were of 34-37 weeks gestational age and $164(78.10 \%)$ were of more than 37 weeks gestational age as shown in Figure 2.

Out of 210 neonates, $48(22.96 \%)$ were born via vaginal delivery while $162(77.14 \%)$ neonates were delivered via Caesarean section as shown in Figure 3. 
Table 1: Distribution according to Birth weight

\begin{tabular}{lccc}
\hline S.No & Weight (Grams) & Frequency & Percentage \\
1. & $1000-1500$ & 2 & $0.95 \%$ \\
2. & $1501-2000$ & 18 & $8.57 \%$ \\
3. & $2001-2500$ & 55 & $26.19 \%$ \\
4. & $2501-3000$ & 97 & $46.19 \%$ \\
5. & $>3000$ & 38 & $18.10 \%$ \\
& Total & 210 & $100 \%$ \\
\hline
\end{tabular}

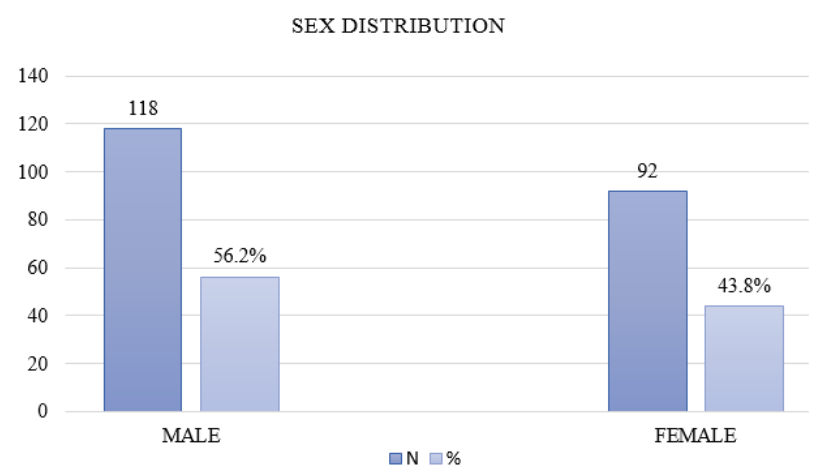

Fig. 1: Sex Distributionof Neonates

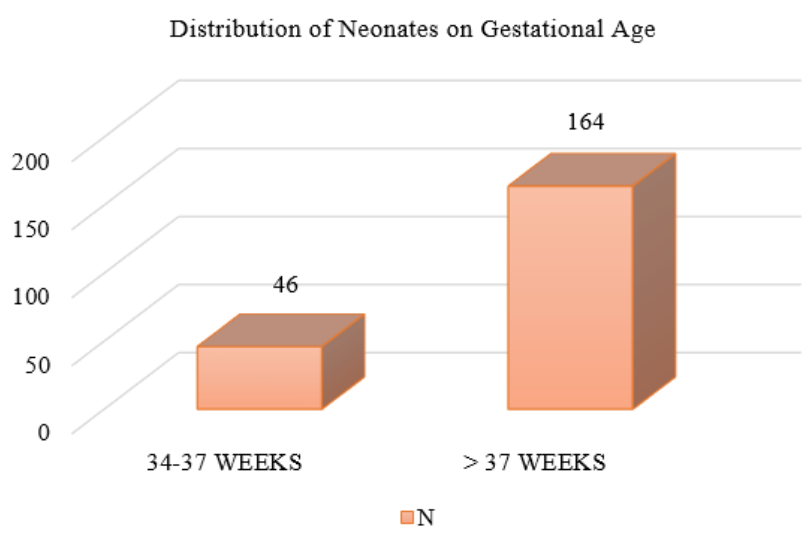

Fig. 2: Distribution Based onGestational Age.

In this study of 210 neonates, $2(0.95 \%)$ were in the weight range of 1000-1500 grams, $18(8.57 \%)$ neonates were between 1501-2000 grams, 55 (26.19\%) neonates were in the 2001-2500 grams range, 97 (46.19\%) neonates were in the 2501-3000 grams range and $38(18.10 \%)$ neonates weighed more than 3000 grams as detailed in Table 1.

Fetal prematurity was found to be the most common risk factor followed by prolonged labor. Birth Asphyxia and PROM $>18$ hours were the other risk factors noted behind the etiologies. as depicted in Table 2 . Considering the Etiology our study have ruled out that out of 210 Neonates studied, the etiology was Idiopathic Hyperbilirubinemia in 28 neonates, physiological hyperbilirubinemia seen in 141

\section{Distribution Based on Mode of Delivery}

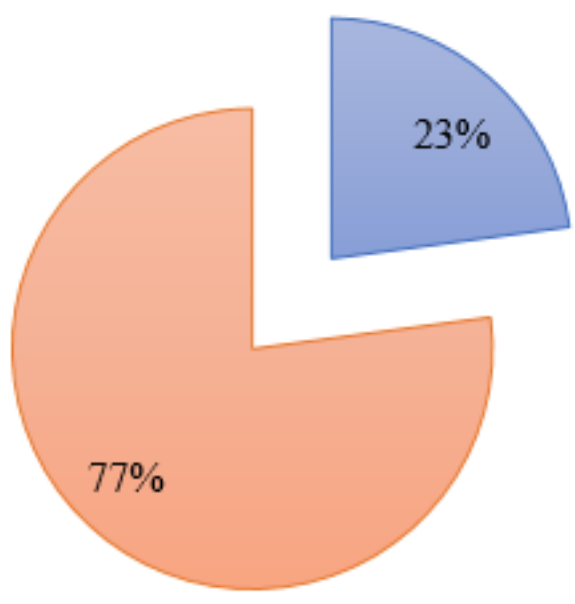

\section{VAGINAL $\square$ C-SECTION}

Fig. 3: Distribution According to Mode of Delivery.

neonates, $\mathrm{ABO}$ and $\mathrm{Rh}$ incompatibility was the cause in 7 neonates each, Septicemia was identified as the cause in 26 neonates and G6PD deficiency was the cause in 1 neonate. Physiological jaundice was taken as Serum Bilirubin levels less than $15 \mathrm{mg} / \mathrm{dl}$ in well infants with no other risk factors for jaundice.

In this study of 210 neonates, at the time of starting phototherapy, 125 (59.52\%) neonates had serum bilirubin between $12-15 \mathrm{mg} / \mathrm{dl}, 42(20.00 \%)$ neonates had bilirubin values between $15.1-18 \mathrm{mg} / \mathrm{dl}$, while $21(10.00 \%)$ neonates had bilirubin values between $18.1-20 \mathrm{mg} / \mathrm{dl}$ and in 22 $(10.48 \%)$ neonates serum bilirubin value was more than 20 $\mathrm{mg} / \mathrm{dl}$ as shown in Table 3 .

From the 210 neonates included in this study, $207(98.57 \%)$ neonates were treated with phototherapy alone while $3(1.43 \%)$ neonates required both exchange transfusion and phototherapy as shown in Table 4 . Rh incompatibility was the etiology behind all $3(1.43 \%)$ neonates requiring exchange transfusion. Intravenous immunoglobulin (IVIG) was not required, as babies were admitted with Bilirubin values in the exchange zones, and warranted an immediate exchange transfusion. Subsequently, there was no alarming rise in bilirubin levels 
Table 2: Distribution based on birth weight

\begin{tabular}{lccccc}
\hline Etiology & "n” & Prom>18 hours & Prolonged Labor & Fetal Pre-maturity & Birth Asphyxia \\
Idiopathic & 28 & 3 & - & 9 & - \\
Physiological & 129 & 3 & 13 & 12 & 4 \\
ABO Incompatibility & 7 & - & - & - & - \\
Rh Incompatibility & 7 & - & - & 3 & - \\
Septicemia & 33 & 2 & 6 & 6 & 3 \\
G6PD Deficiency & 1 & - & - & 30 & - \\
Total & 210 & 8 & 19 & & 7 \\
\hline
\end{tabular}

Table 3: Distribution Based on Sr. Bilirubin when starting Phototherapy

\begin{tabular}{|c|c|c|c|}
\hline S. No & Sr. Bilirubin level (mg/dl) & Frequency & Percentage \\
\hline 1. & $12-15$ & 125 & $59.5 \%$ \\
\hline 2. & $15.1-18$ & 42 & $20 \%$ \\
\hline 3. & $18.1-20$ & 21 & $10 \%$ \\
\hline \multirow[t]{2}{*}{4.} & $>20$ & 22 & $10.4 \%$ \\
\hline & Total & 210 & $100 \%$ \\
\hline
\end{tabular}

Table 4: Distribution of Neonates Based on Mode of Therapy

\begin{tabular}{|c|c|c|c|}
\hline S.No & Mode of Therapy & Frequency & Percentage \\
\hline 1. & Phto therapy Alone & 207 & $98.5 \%$ \\
\hline 2. & Exchange Transfusion and Photo Therapy & 3 & $1.43 \%$ \\
\hline Total & & 210 & $100 \%$ \\
\hline
\end{tabular}

Table 5: Complications Developed During Treatment.

\begin{tabular}{lc}
\hline Complications during phototherapy & Number of patients \\
Loose motions & 4 \\
Rash & 3 \\
Total & 7 \\
Complications during exchange transfusion & Number of patients \\
Cardiac arrest & 0 \\
Hypoglycemia & 0 \\
Septicemia & 0 \\
Total & 0 \\
\hline
\end{tabular}

and did not warrant IVIG therapy

During phototherapy, 4 neonates developed loose stools while 3 neonates developed rashes. There were no complications seen during exchange transfusion for all 3 neonates as shown in Table 5.

In our study, the average BIND score for babies with Idiopathic pathological jaundice was 3 , for $\mathrm{ABO}, \mathrm{Rh}$ incompatibility and Septicemia the score was 2 and the average score for babies with Physiological jaundice and due to G6PD deficiency was 1. An average BIND score of 1.8 was thereby noted for the 210 neonates involved in this study as shown in Figure 4.

\section{Discussion}

Total of 210 neonates of neonatal hyperbilirubinemia were studied in detail

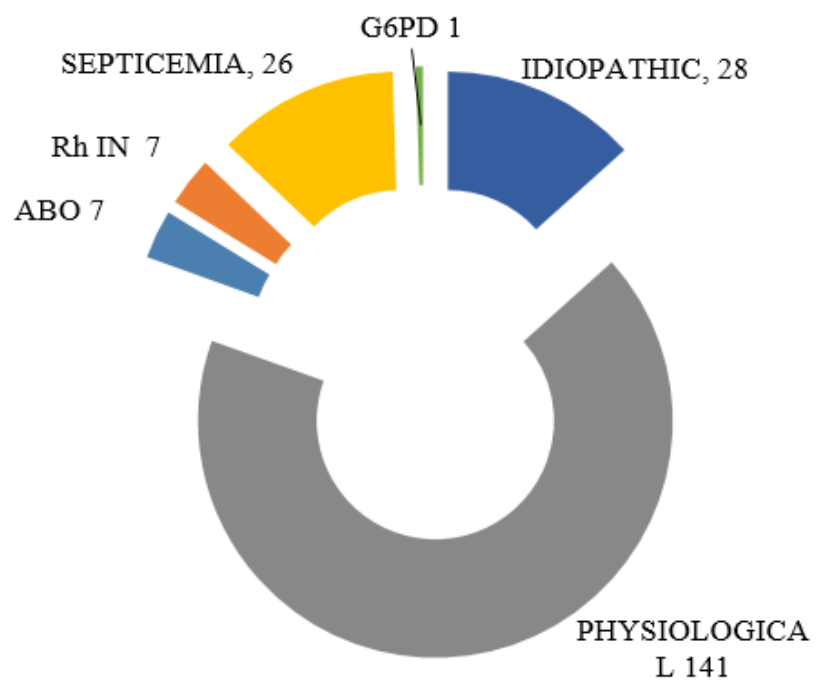

Fig. 4: Distribution based on BIND Score and Etiology. 


\subsection{Sex distribution}

Out of 210 neonates, $56.20 \%$ were males and $43.80 \%$ were females.

Narang et al $^{12}$ also found a male predominance in their studies with 56.2 cases of the male sex.

\subsection{Gestational age}

46 neonates from the 210 were pre-term with $21.90 \%$ incidence while 164 neonates having an incidence of $78.10 \%$ were more than 37 weeks. Singhal et al ${ }^{13}$ have found prematurity as the second most common cause of hyperbilirubinemia in their studies. Narang et al $^{12}$ found jaundice in $47.9 \%$ out of 917 preterm babies.

\subsection{Age at admission}

From the 210 babies involved in this study, the average age of the babies at the time of admission for phototherapy was 5 days of life.

\subsection{Birth weight}

In our study, maximum number of neonates (97) were having birth weight between 2501-3000 grams. $2(0.95 \%)$ neonates were below 1500 grams and $18(8.57 \%)$ neonates were between 1501 and 2000 grams. 38 (18.10\%) neonates had birth weight more than 3000 grams. Singhal et al ${ }^{13}$ found in his study that one-fourth of neonates $(29.16 \%)$ developing hyperbilirubinemia were with low birth weight.

Narang et al $^{12}$ concluded from their studies that the incidence of neonatal jaundice was three times higher in LBW babies compared to babies above 2500 grams.

In our study, $35.71 \%$ neonates were of low birth weight. It is mainly because of maternal under nutrition, poverty, underutilization of health services and illiteracy seen in the general area.

\subsection{Risk factors}

1. Maternal: In our study maximum number (19) of babies who developed jaundice had prolonged labor while 8 neonates had PROM more than 18 hours.

2. Fetal: In 30 neonates prematurity was the aggravating factor while 7 neonates had birth asphyxia. Singhal et $\mathrm{al}^{13}$ found prematurity as a cause for jaundice in $16.7 \%$ neonates. They have also found birth asphyxia as aggravating factor for hyperbilirubinemia in $12.1 \%$ cases out of which maximum were in idiopathic group. Our findings correlate with Singhal et al. ${ }^{13}$

\subsection{Etiology}

1. Physiological hyperbilirubinemia: In 141 neonates, the jaundice was physiological as it appeared between 48-96 hours and disappeared by 6-7 days in term and
10-12 days in preterm babies. Our findings are similar to Merchant et al ${ }^{14}$ and the findings in their study.

2. Intervention with phototherapy was started at bilirubin levels of $12 \mathrm{mg} / \mathrm{dl}$ as very often, parents do not report for close follow-up post discharge and often are referred late in the course of illness with pathological jaundice.

3. Idiopathic hyperbilirubinemia: In 28 (13.33\%) neonates, the cause was unidentified and labelled as Idiopathic.Merchant et $\mathrm{al}^{14}$ found idiopathic hyperbilirubinemia in 66\% cases, Verma M. et al[15] found idiopathic in $35 \%$ cases, Singhal et al ${ }^{13}$ found idiopathic as the cause in $34.4 \%$ cases while Narang et $\mathrm{al}^{12}$ found it in $57.8 \%$ cases. Our findings are close to that of Verma M. et al[15] and Singhal et al. ${ }^{13}$

4. Septicemia: In 26 (12.40\%) neonates, septicemia was the cause for jaundice out of which 14 were preterm and 19 were term babies. Various aggravating factors like PROM $(n=2)$, Prolonged labor $(n=6)$, Prematurity $(n=6)$ and Asphyxia $(n=3)$ were seen.

5. Various laboratory investigations were suggestive of septicemia with total WBC count $>15,000$ and elevated CRP $(>6)$ values. Blood culture was positive in 15 neonates. All the neonates were treated with appropriate antibiotics. Septicemia as a cause for hyperbilirubinemia was found in $8 \%$ neonates by Merchant et al, ${ }^{14}$ in $11.6 \%$ neonates by Verma et al,[15] in $5.7 \%$ neonates by Singhal et $\mathrm{al}^{13}$ and in $9.6 \%$ neonates by Narang et al. ${ }^{12}$ Our findings are in accordance with Verma et al[15] and Narang et al. ${ }^{12}$

6. Rh incompatibility: This was noted in 7 (3.33\%) neonates. Direct Coomb's test was positive in all these neonates. Other investigations revealed hemolytic picture on peripheral smear and high reticulocyte count. $\mathrm{Rh}$ incompatibility was found in $18.6 \%$ neonates by Merchant et al, ${ }^{14}$ in $8.1 \%$ neonates by Singhal et al, ${ }^{13}$ in $2.9 \%$ by Narang et al, ${ }^{12}$ and in $9.6 \%$ by Verma M. et al.[15] Our findings are close to Narang et al. ${ }^{12}$

7. $\mathrm{ABO}$ incompatibility: Incidence of $\mathrm{ABO}$ incompatibility was same as that of $\mathrm{Rh}$ incompatibility at $3.33 \%$. Merchant et al ${ }^{14}$ found $\mathrm{ABO}$ incompatibility in $22.6 \%$ neonates, Verma $M$. et al[15] found it in $22.6 \%$ and Singhal et $\mathrm{al}^{13}$ in $14.3 \%$ neonates. Our findings are closest to Singhal et al. ${ }^{13}$

8. G6PD deficiency: It was found in only one neonate. Baby developed jaundice between 48-72 hours. G6PD deficiency was found in $2.6 \%$ neonates by Merchant et al, ${ }^{14}$ in $5.1 \%$ by Singhal et al ${ }^{13}$ and in $3.4 \%$ neonates by Narang et al. ${ }^{12}$ Our findings are closest to Merchant et al. ${ }^{14}$ The prevalence of G6PD deficiency is uncommon in Southern India. 


\subsection{Therapeutic interventions}

Out of the 230 neonates, 3 required exchange transfusion. All of these 3 neonates had $\mathrm{Rh}$ incompatibility. Exchange transfusion was done when the serum bilirubin concentrations were in the transfusion zone according to the AAP charts for exchange transfusion. None of the babies developed BIND. Other risk factors like birth asphyxia, septicemia, hypoglycemia and acidosis were also considered while deciding exchange transfusion.

Singhal et al. ${ }^{13}$ found in their study that the commonest etiology behind babies requiring exchange transfusion was Rh incompatibility followed by ABO incompatibility and G6PD deficiency. Narang et al $^{12}$ found that the most common cause was Idiopathic, followed by Septicemia and G6PD deficiency. Rest of the 207 neonates in our study received phototherapy alone. Phototherapy was started when serum bilirubin was more than $12 \mathrm{mg} / \mathrm{dl}$ and was given for 24,48 or 72 hours.

\subsection{Side effects}

In our study, due to phototherapy, 4 neonates developed loose stools while 3 neonates developed a rash all over the body. There were no side effects seen as a result of exchange transfusion in any of the babies.

\subsection{BIND scoring}

An average BIND score of 1.8 was noted among the 210 neonates included in this study. None of the neonates developed encephalopathy. This is probably due to early identification and referral of these infants by their pediatricians for early intervention.

\section{Conclusion}

1. Most common cause of neonatal hyperbilirubinemia was found to be Physiological followed by Septicemia and Idiopathic etiologies. Blood group incompatibilities were less common causes.

2. Photo therapy is a cheap and effective way to reduce bilirubin levels in neonatal jaundice.

3. Need for exchange transfusion has significantly reduced due to more awareness and cautiousness of treating pediatricians. Exchange transfusion is a safe procedure and should be considered when indicated, to decrease the incidence of BIND. 11

\section{Conflict of Interest}

The authors declare that there are no conflicts of interest in this paper.

\section{Source of Funding}

None.

\section{References}

1. Avery GB, Maisels MJ, Jaundice. Pathophysiology and management of newborn. In: 5th Edn. Philadelphia: Lippincott JB Company; 1999

2. Gleason C, Ballard R. Avery's Diseases of the Newborn. In: 8th Edn. Philadelphia Elsevier; 2005.

3. Anand VR, Magotra ML. Neonatal jaundice: its incidence and aetiology. Indian Pediatr. 1978;15(2):155-60.

4. Bahl L, Sharma R, Sharma J. Etiology of neonatal jaundice at Shimla. Indian Pediatr. 1994;31(10):1275-8.

5. Behrman RE, Hsia DYY. Summary of a Symposium on Phototherapy for Hyperbilirubinemia. J Pediatr. 1969;75(4):718-26. do1:10.1016/S0022-3476(69)80475-0.

6. Johnson L, Bhutani VK. Guidelines for Management of the Jaundiced Term and Near-Term Infant. Clin Perinatol. 1998;25(3):555-74. do1:10.1016/s0095-5108(18)30097-6.

7. Narang A, Gathwala G, Kumar P. Neonatal jaundice: an analysis of 551 cases. Indian Pediatr. 1997;34(5):429-32.

8. Lochmann KK, Sodhi M, Singh G. Incidence of neonatal jaundice. Abstracts Pedicon. 2004;p. 158-9.

9. Agarwal R, Deorari AK. Unconjugated hyperbilirubinemia in newborns: current perspective. Indian Pediatr. 2002;39:30-42.

10. Misra PK, Govil YC. Neonatal Hyperbilirubinemia. IAP J Practical Paediatr. 1994;2(4):361-66.

11. Dennery PA, Rhine WD, Stevenson DK. Neonatal Jaundice - What Now? Clin Pediatr. 1995;34(2):103-7.

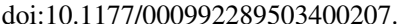

12. Narang A, Gathimala G, Kumar P. Neonatal Jaundice: An analysis of 551 cases. 1997;34:429-32.

13. Singhal PK, Singh M, Paul VK, Deorari AK, Ghorpade MG. Spectrum of neonatal hyperbilirubinemia: an analysis of 454 cases. Indian Pediatr. 1992;29(3):319-25.

14. Merchant RH, Merchant SM, Babar ST. A study of 75 cases of neonatal jaundice. Indian. Indian Pediatr. 1975;12(9):889-93.

\section{Author biography}

Syed Adnan Ali, Resident

C.V.S. Lakshmi, Assistant Professor

Uppin Narayan Reddy, Professor

Farhana Nazneen, Resident

Muzammil, Resident

Cite this article: Ali SA, Lakshmi CVS, Reddy UN, Nazneen F,

Muzammil. A prospective study on etiological factors and clinical indicators in term and near term neonates admitted with

hyperbilirubinemia in nicu in a tertiary care hospital in Hyderabad, India. IP Int J Med Paediatr Oncol 2021;7(2):51-56. 